\title{
Como comunicamos las/os enfermeras/os: una reflexión necesaria
}

Autora:

Cibeles González Nahuelquin. Enfermera. Magíster en Enfermería. Profesor Adjunto Escuela de Enfermería. Universidad de Valparaíso, Chile. E- Mail: cibeles.gonzalez@uv.cl.

\section{> Resumen}

La comunicación es un proceso dinámico y fluido, que se genera a partir de la relación que se establece con el otro. Para el profesional de enfermería esta competencia se transforma en un vínculo vital que le permite generar una relación armónica y confiable en el proceso de cuidado. Sin embargo, la vorágine en el quehacer profesional, favorece a que tanto la relación como la comunicación que se instaura con la persona cuidada, corra el riesgo de deshumanizarse. El siguiente manuscrito tiene como propósito, reflexionar sobre como es el proceso de comunicación en la disciplina enfermera, entregando herramientas que permitan fortalecer la relación interpersonal y con ello llevar a repensar e inspirar a la enfermería con la base de su esencia, que es el cuidado humano.

> Palabras clave: comunicación, comunicación en salud, atención de enfermería, humanización de la atención (DeCS) 


\section{How nurses communicate:}

a necessary reflection

\section{> Abstract}

Communication is a dynamic and fluid process, which is generated from the relationship established with the other. For the nursing professional this competence becomes a vital link that allows him to generate a harmonic and reliable relationship in the care process. However, the vortex in the professional work, favors that both the relationship and the communication that is established with the person cared for, have the risk of dehumanizing. The main purpose of the following manuscript is to reflect on the communication process in the nurse discipline, providing tools to strengthen the interpersonal relationship and thus lead to rethink and inspire nursing with the basis of its essence, which is the human care.

> Keywords: Communication, health communication, nursing care, humanization of assistance (DeCS) 


\section{) Introducción}

La palabra comunicación tiene su origen en el latin "comunicare", que significa poner en común (1). Para colocar alguna cosa en común, es necesario que haya el entendimiento y comprensión de lo que se comunica en la relación que se establece (2). De esta forma, el ser humano se transforma en una persona por naturaleza sociable, entregando a través del intercambio de mensajes, sentimientos, emociones, costumbres y opiniones, lo que conlleva que la comunicación cumpla un papel esencial en todos los procesos de la vida (3).

El proceso de comunicación en la disciplina enfermera cumple un papel indispensable, ya que es en el cuidado donde se refleja el más genuino acto de humanidad, la confianza y afectividad mutua de la relación que se establece con la persona cuidada, permitiendo que la/el enfermera/o y el paciente establezcan una relación armoniosa y por ende una comunicación fluida, la cual se espera favorezca la recuperación de la salud y el bienestar de la persona $(4,5)$.

La comunicación que el profesional enfermero establece con la persona que atiende es un proceso sustentado en la experiencia de reconocer y entender lo que ella /él siente, piensa y hace, reaccionando de manera empática (5). De esta manera, el intercambio de mensajes que realiza el profesional es una herramienta que permite no solo favorecer la comprensión, aceptación y la operatividad del tratamiento, sino también es una herramienta eficaz que ayuda a disminuir el sufrimiento (4).

De esta forma, comunicarse para una/un enfermera/o es parte de su esencia, parte del cuidado, parte de la relación que establece con el otro. Henderson, describió que este tipo de acción, es un proceso interpersonal, donde se desarrolla la empatía, refiriendo textualmente que la enfermera "debe meterse en la piel de sus pacientes", a fin de conocer que ayuda necesitan (6).

Así, la/el enfermera/o, debe obligatoriamente establecer la comunicación como un eje articulador del cuidado que entrega, mostrando la cercanía con el paciente por medio de expresiones verbales y no verbales, lo cual favorecerá la aplicación de cuidados técnicos y propiciará no solo la satisfacción de la persona que se atiende, sino también la satisfacción de ella /el como profesional (7).

Considerando los antecedentes entregados, el siguiente artículo se ha propuesto reflexionar sobre la importancia que tiene el proceso de comunicación en la relación de la enfermera - paciente. Para comenzar, se contextualiza el proceso de comunicación, posteriormente se analiza su importancia en la enfermería y finaliza con una construcción teórica, que implica herramientas que le permitan al profesional de enfermería tener una comunicación efectiva, terapéutica, interpersonal y empática con la persona, familia o comunidad a la cual entrega su cuidado.

\section{LA NECESIDAD DE COMUNICARSE}

Desde tiempos remotos el hombre ha tenido la necesidad de comunicarse, de expresar sus sentimientos a otro, de ser escuchado y comprendido (8). Esto lo observamos en escritos, pinturas y/o grabados que nos han legado pueblos ancestrales, donde por medio de ellos, demuestran la necesidad de establecerse en grupos, de ejercer liderazgo y de comunicarse (8). En Chile, por ejemplo, la historia rescatada del pueblo mapuche, revela la importancia que tenía - y que aún conserva- para esta etnia, la comunicación, esta se encontraba relegada principalmente a la oralidad, es decir, al traspaso de conocimientos, de creencias y de valores a través del lenguaje hablado de generación 
en generación, acción que ha sido fundamental para mantener la cultura y cosmovisión a través de los años (9).

De esta forma, tomando en consideración la tradición que nos antecede, se afirma que las formas más comúnmente adaptadas por el hombre para poder establecer una relación con el otro, es la expresión verbal y la escritura. Si bien, la primera depende de la segunda y esta a su vez de la anterior, cada una posee cualidades diferentes, que hacen que la comunicación sea un proceso fluido, integrador, interactivo, continuo y modificable $(8,9)$.

Cuando se habla del proceso de comunicar, se habla de una interacción social, donde se transmite información, se intercambian ideas, necesidades, costumbres y toda clase de opiniones y pensamientos entre un individuo que emite un mensaje y otro que lo recibe. Así, el proceso va conformando la base del desarrollo psíquico, psicológico, social y cultural de los seres humanos (10).

Para que este proceso sea eficaz - constructivo, comprendido y fluido- debe haber una intención y un contacto entre aquellos que establecen la relación, donde el intercambio de mensajes debe contener algunos elementos importantes y necesarios: un emisor, el cual es la fuente y origen de donde procede lo que se quiere comunicar; un receptor, quien recibe la información; un código, el cual corresponde a un conjunto de signos que se utilizan para transmitir el mensaje y un canal de comunicación, que corresponde a un medio fisico por el cual el emisor hace llegar el mensaje al receptor $(2,11)$.

Al establecer una relación adecuada entre estos elementos y de acuerdo a la importancia que adquieren unos u otros, se comienza a hablar de las funciones del lenguaje, estas son: la función emo- tiva, apelativa, referencial o informativa, poética, metalingüística y la función situacional, con ellas se logra la interacción comunicativa (11).

Cuando se construye una interacción comunicativa, se establecen formas y modos de comunicación, logrando así una red coherente de mensajes enviados y recibidos, es decir, se logra un patrón comunicativo que define la forma más o menos habitual en que nos relacionamos (11), en el caso de la enfermería, como el profesional se relaciona con el paciente y su familia.

Todos los modelos comunicativos señalan que este proceso dinámico, posee dos elementos interactuantes principales: 1) el valor del contenido del mensaje y 2) el valor interaccional o perceptual del mensaje y de sus participantes, también llamado metacomunicación. El primero se refiere al aspecto de la información en si y el segundo hace referencia a como se interpreta el contenido y la forma en que los participantes perciben la relación, ambos pueden ser de tipo verbal y no verbal $(2,12)$. Entonces, la comunicación no solo se relaciona a la palabra hablada y al uso de mecanismos fisiológicos y cognoscitivos funcionales para hacer posible la producción y recepción del mensaje, sino que se relaciona también a esos aspectos no verbales que facilitan la percepción más exacta de los sentimientos entre dos o más personas $(2,12)$, caracterizándose por una interacción continua entre quienes participan en él, donde emisor y receptor se influyen mutuamente. Este fenómeno, se conoce como el principio de la reciprocidad, fue descrito por primera vez en la década del 60' y se ha constituido en la base de la comunicación y de la relación interpersonal del proceso de enfermería (12).

Por tanto, la necesidad de comunicarse es la base fundamental de las relaciones humanas, desempeña el papel más importante a la hora de ma- 
nifestar los sentimientos, emociones, formas de pensamientos, cultura y creencias, por lo que es posible aseverar que la comunicación facilita la comprensión del otro y de su forma de ver el mundo $(2,4,7-9)$.

\section{LA IMPORTANCIA DE LA COMUNICACIÓN PARA EN- FERMERÍA}

La competencia de la comunicación para la/el enfermera/o tiene diferentes acepciones, una de ellas la define como "la capacidad para transmitir hábilmente, de manera eficaz y oportuna opiniones, intenciones y creencias sin provocar deterioro en la relación con los demás" $(13,14)$. Así defini$\mathrm{da}$, se enmarca conceptualmente con actividades desempeñadas especialmente en el área de la gestión clínica.

Otros autores afirman que la comunicación en enfermería es mayormente interpersonal, colocando en esta un sello humanizado, donde existe una interacción permanente entre el profesional y la persona que se atiende. Esta interacción debe mostrar comprensión, respeto y reciprocidad, incorporando y adaptando elementos de la escucha activa y del lenguaje a la propia idiosincrasia, lo que es percibido por las personas como un involucramiento genuino de la/el enfermera/o en su atención (4).

Si bien, ambas acepciones son utilizadas en la disciplina enfermera, la segunda adquiere una mayor validez a la hora de establecer una relación terapéutica, armónica e interpersonal con la persona que se cuida, puesto que, permite el adecuado reconocimiento de sus necesidades y por ende, la pronta recuperación de su salud $(2,5,15)$.

Entonces, tomando en consideración lo anterior, la comunicación para la enfermería, corresponde al primer vínculo que se establece con el paciente y su familia en el proceso de cuidar, se transforma en un elemento vital en la calidad de la relación, en la identificación del continuo salud-enfermedad y en la realización del cuidado propiamente tal, transformándose en un proceso recíproco, donde ambos (enfermera/o- paciente) se benefician $(2,16)$.

La hospitalización o el enfrentamiento a un sistema de salud - cualquiera sea su complejidad- genera un estrés en la persona o familia, por lo que entregar una adecuada orientación, la cercanía y la comunicación que preste el profesional de enfermería genera sentimientos de confianza (15). Sin embargo, este proceso muchas veces se ve disminuido o incluso ausente, debido a que actualmente se entrega mayor importancia al conocimiento teórico y técnico que al fortalecer la relación con el otro, generando una forma de despersonalización y deshumanización en la atención, teniendo así una comunicación, mayormente de tipo referencial e informativa (4,16-19,24).

Dicha situación ha sido planteada Jean Watson, señalando que la enfermería se encuentra en un proceso ambiguo en esta área, donde se esta permitiendo que la ciencia prime en la entrega de cuidados, relegando y postergando importancia al aspecto psicosocial, que es la base de la disciplina enfermera $(6,18)$. Se rescata textual de su apreciación: "Enfermería ha perdido su alma por servir ya sea a la medicina, la tecnología y la práctica basada en la evidencia, las que por cierto se necesitan; pero sin la dimensión humana, esto puede ser destructivo. Es fundamental preservar la esencia humana y reposicionar el cuidado dentro de nuestros sistemas" (18).

Esta reflexión, no solo despierta el interés por analizar aspectos inherentes a la comunicación para enfermería (en la relación persona-persona), sino que también despierta el interés en identificar herramientas que permitan mejorar la calidad 
de esta comunicación, con el fin de perfeccionar la tensión existente en los discursos dominantes, tales como la tecnología, la enfermedad, la práctica basada en la evidencia y la enfermería psicosocial $(12,18)$.

Así, la comunicación para la/el enfermera /o no es solo un proceso, sino un recurso que permite al profesional establecer una relación interpersonal con quien ayuda, es una condición sine qua non para ofrecer cuidados de calidad y asertivos que permitan fortalecer el autocuidado y la autonomía del otro (20).

Para lograr la autonomía de la persona que se cuida, el profesional en su proceso de comunicación, establece ciertos aspectos que influyen de manera directa en la atención que se entrega, los cuales son (12,21):

Patrón de relación social convencional: se refiere a las relaciones sociales convencionales, a reglas dictadas por la costumbre o porque se considera un comportamiento apropiado, por lo tanto, para poder comportarse convencionalmente, el individuo necesita conocer esas reglas y debe ser capaz de llevarlas a la práctica. Obtener tal conocimiento dentro de la disciplina enfermera, influye directamente en el quehacer del profesional, puesto que, al mantener un comportamiento socialmente aceptable o profesional con el paciente, familia y/o comunidad en la cual trabaja, facilitará la obtención de resultados positivos en la intervención que se realiza o que se quiera realizar. Sin embargo, mantener siempre este tipo de patrón, puede favorecer una interacción comunicacional superficial, porque no permite que ninguno de los participantes conozca al otro como ser humano único, y si la/el enfermera/permanece en este nivel de comunicación, es probable que entorpezca el comportamiento esperado y no brinde la ayuda necesaria para que el paciente logre alcanzar la recuperación de su salud.

Patrón de información o utilitario: se refiere a la forma de comunicarse de un individuo en forma imperativa, es decir, da instrucciones, mandatos u órdenes a los demás. Este patrón es ventajoso cuando la información enviada es útil para el que la recibe. En la disciplina enfermera, se utiliza en indicaciones de cuidados básicos, como por ejemplo, la realización del lavado de manos previo a la atención directa, sin embargo, también es posible observarlo en la entrega de contenidos educativos, en donde la intervención se transforma en una cadena comunicativa dirigida unilateralmente. Por lo tanto, es de responsabilidad de la/ el enfermera/o identificar aquellas intervenciones que deshumanizan el cuidado y ser agente de cambio en los cuidados que brinda.

Relacionarse como patrón de comunicación: Es la experiencia o serie de experiencias que se caracterizan por un diálogo significativo entre dos individuos. En el caso de la enfermería, el diálogo enfermera/o-paciente, donde cada uno comprende la sinceridad, la unión y el entendimiento con el otro por medio de una comunicación recíproca, la cual se obtiene al conocer al otro, al usar el conocimiento en beneficio del otro, a la sensibilidad y a la oportunidad de la interacción recíproca. Así, el profesional de enfermería debe estar dispuesto a permitir que coexistan problemas o sentimientos opuestos, con el fin de estructurar una interacción significativa, de modo que una persona pueda sentir que sus problemas son escuchados y que existe una interacción comunicacional, cuya finalidad es apoyarlo en la búsqueda y encuentro de la recuperación de su salud.

El diálogo entre los diferentes patrones, permite al profesional de enfermería establecer una relación reciproca e interpersonal y por ende, una comunicación asertiva, relevante e interactiva a 
fin de alcanzar los objetivos que se tienen con el cuidado.

\section{CONSTRUCCIÓN TEÓRICA PARA UNA COMUNICA- CIÓN EFECTIVA EN ENFERMERÍA}

Para que la/el enfermera/o pueda despojarse de todos los prejuicios y logre establecer una comunicación efectiva, asertiva, interpersonal y humanizada con la persona que atiende, debe volver a la esencia de la enfermería, a recordar que el cuidar fundamenta el Ser Enfermero/a, donde cada profesional "es" en la relación con el otro, reconociéndolo en toda su forma como una persona única, capaz de ejercer su autonomía y libertad (en mayor o menor grado). Esto se logra al comprender que el paciente no le pertenece, incluso en estados de mayor vulnerabilidad $(6,18)$.

Incorporando lo señalado, el profesional debe ser capaz de comunicarse efectivamente, pero ¿cómo lograr que este fundamento teórico atraviese y permee al conocimiento práctico, tan en boga en este tiempo?

Para ello, se necesita que la/el enfermera/o posea competencias y habilidades que le permitan fortalecer y mejorar la comprensión del otro. En este sentido, la filosofía propuesta por Jean Watson, postula que los cuidados deben ser transpersonales, esto significa "tener relación de cuidado humana- una unión con la otra persona- muy respetada para la persona y su estar en el mundo", para lo que establece diversos factores que permiten a la/el enfermera/o mantener una relación y comunicación verdadera, fluida, humanizada y real $(6,18,22)$.

Para establecer una relación y comunicación armónica entre lo que describe Watson en su filosofía y lo que requiere la/el enfermera/o en su práctica diaria, se proponen los siguientes constructos, que se esperan, permitan fortalecer esta competencia:

1. Respetar la fe a través de la verbalización, tanto del profesional como de la persona enferma: esta habilidad esta basada en la capacidad de mantener un sistema de creencias y valores, que sean compatibles con la libertad de cada uno de los involucrados en el proceso comunicativo. Para la persona que es cuidada la verbalización disminuye la tensión y la ansiedad frente a la patología que lo aqueja, el hablar no solo de su enfermedad, sino de sus pensamientos, temores y dudas respecto a lo que le acontece, le ayuda a sobrellevar la experiencia de la enfermedad, el creer en "algo" o "alguien" genera una forma de confianza y el verbalizar este sentimiento contribuirá al proceso de sanación $(6,12,18)$. Para el profesional, la verbalización le capacita para identificar las necesidades de la persona enferma y en el sentido de la fe y la esperanza, le permite generar un ambiente de confianza en el cuidado que se brinda, lo describe así un pensamiento alojado en la memoria del colectivo de enfermería "no siempre es factible curar, pero siempre es posible cuidar", la fe no necesariamente logrará la curación, pero siempre estará asociada al cuidado $(18,23)$.

\section{Ser sensible consigo mismo y con los demás:} este aspecto pretende comprender las dinámicas personales, sociales y culturales de cada uno de los involucrados en el proceso comunicativo. Watson señala que "el ser humano es un ser sintiente", por lo que, es necesario que el profesional de enfermería en su quehacer, no separe su sensibilidad hacia el otro, puesto que, si esto sucede, las personas (o pacientes) tenderán a esconder, negar o disimular sus sentimientos $(6,18,23)$. Cuando la / el enfermera/o se rehúsa a reconocer sus propios sentimientos, dificilmente podrá comprender los sentimientos del otro (18), el demostrar los sentimientos y ser honestos con ellos, permitirá y fa- 
cilitará la comunicación que se establezca en la relación enfermera/o-paciente y su familia.

\section{Demostrar empatía y bondad en el contexto} del cuidado: este aspecto se genera a través de la práctica amorosa, donde el profesional de enfermería reflexiona desde su propio desarrollo moral, desde su propia experiencia y del desarrollo que ha logrado como individuo $(6,18)$. El demostrar empatía en el proceso comunicativo con la persona que se atiende, evidencia la capacidad de participar en los sentimientos o ideas del otro, sintiendo, compartiendo y aceptando aquellas emociones. Demuestra estar conciente del carácter único de la persona, considerándolo como en ser igual a ella/el misma/o, es decir, si el paciente se percata de que es tratado como un "objeto" se pondrá de inmediato a la defensiva, sin embargo, si percibe que la/el enfermera/o se interesa por él y por lo que siente, se generará una relación de confianza, reflejada en la comunicación que establezca con ella/el (6,12).

Para que el profesional de enfermería desarrolle esta empatía, debe poseer dos condiciones, la primera es aceptarse a sí mismo como persona y, la segunda es poseer la capacidad de escuchar todos los mensajes transmitidos por el paciente, siendo sensible y respetuoso con cada uno de ellos $(6,12)$. Si la/el enfermera/o ejercita este tipo de acción, logrará una comunicación eficaz $(6,12,18)$.

\section{Desarrollo de una relación de ayuda y con-} fianza: para su desarrollo es necesario que la/el enfermera/o fortalezca no solo la sensibilidad y el altruismo sino que es de vital importancia que fortalezca la congruencia, la honestidad y la verdad.

Para Watson, este último se logra delimitando tres guías (18):

- Evitando la emisión de discursos programados tipo “contestador telefónico", los cuales pue- den bloquear la autenticidad y favorecer la distancia del y para el paciente.

- Reconocer las dificultades propias de la relación que se establece con el otro, siempre recordando, que el proceso comunicativo posee dos emisores y dos receptores.

- Reconocer que las experiencias que tienen los pacientes y la/el enfermera/o son vitales para el proceso comunicativo, lo cual se basa en el autoaprendizaje y la introspección.

\section{Fortalecer y mantener la creatividad del "arte} de cuidar": La concepción estética de la enfermería, de donde proviene el constructo "arte de cuidar", es una dimensión que representa la belleza de la profesión. Nightingale ya lo describía en 1959, donde enfatiza como arte al "proceso de la imaginación creativa, el espíritu sensible y la comprensión inteligente" que fundamenta las acciones concretas del cuidado, concepto que rescata la dimensión esencial de la naturaleza de la enfermería (25). El mantener la creatividad y la belleza de la disciplina enfermera en el proceso comunicativo, es un desafío constante, es un aspecto que se tiene que sobreponer a la multiplicidad de actividades que realiza la/el enfermera/o en su quehacer diario y sobre todo a la rigidez y mecanicidad en las acciones, que si bien permiten la resolución de los problemas, impiden visualizar el fondo de la acción, de la relación y de la comunicación que se establece con el otro. En este sentido, para Watson, es necesario disminuir o eliminar la sistematización excesiva de normativas, los cuales impiden el desarrollo creativo de la profesión, recordando que cada individuo desarrolla su forma de encontrar significados en la vida (18).

Lo expuesto, fija lineamientos o guías para que el profesional de enfermería pueda establecer una comunicación efectiva, que si bien se encuentra 
implícita en la profesión y en el cuidado, es necesario valorar, fortalecer y mejorar, para generar una nueva visión, donde el cuidado se brinde en base a las necesidades sentidas de los pacientes (15).

El mantener una actitud efectiva y afectiva en el cuidado, supone un conocimiento de lo que los pacientes necesitan, donde la personalización, la humanización, la actitud cercana y respetuosa hacia el otro y las habilidades comunicativas deben ejercer un papel fundamental, recordando siempre cual es la esencia de la profesión, que no es otro que el servir, lo que debe realizarse con amor.

Para finalizar, es importante recodar lo señalado por Watson, para quien el cuidado de enfermería se caracteriza por su naturaleza intencional y se funda en el conocimientos y destrezas específicas complejas, ella describe (el cuidado) como el "proceso... de compromiso conciente que armoniza con el amor a nivel universal y por ello tiene un alto potencial sanador, cuyos efectos se extienden más allá de los límites del acto de cuidado" (25).

\section{CONSIDERACIONES FINALES}

El proceso de comunicación es un proceso dinámico, con un continuo intercambio de mensajes, transformándose así, en un fenómeno interindividual, interno - externo e individual - colectivo, donde la comprensión y la interpretación de cada emisión y recepción de mensajes posibilita innumerables significaciones (2).

La comunicación para la/el enfermera/o constituye un pilar fundamental en el proceso de cuidado, le permite encontrar en aquellas significaciones recibidas, la información necesaria para identificar las necesidades y establecer un proceso de cuidado $(4,15,22,23)$, que tiene por finalidad ayudar al paciente y su familia a asimilar su problema de salud y eventual tratamiento, estableciendo así el carácter humano de la disciplina (22).

Establecer un modelo de comunicación específico para la disciplina enfermera, es un desafio, ya que debe involucrar aspectos científicos y humanistas que permitan un sincretismo no competitivo entre sus componentes, donde generalmente, el elemento científico cobra mayor relevancia a hora de entregar los cuidados (4).

Actualmente existen teorías que acercan a la/el enfermera/o a la esencia de la disciplina, donde se establece que la humanización es un componente significativo y único en el quehacer del profesional, donde el proceso de comunicación cobra relevancia para conocer al otro, para posicionarse en su realidad y comprenderlo.

Lo expuesto hace reflexionar sobre la manera de comunicarnos como enfermeras/os, sobre la importancia que le damos a esta competencia y como podemos establecer herramientas que nos permitan mejorar y fortalecer la habilidad comunicativa en el proceso enfermero.

Se espera que esta reflexión permita inspirar a los profesionales de enfermería, a re-pensar cómo es nuestro quehacer diario, cómo establecemos modelos de comunicación, que muchas veces son imitados por las nuevas generaciones, cómo estamos entregando el cuidado y cómo es la relación con el otro.

Para finaliza, se recuerda la frase inspiradora de esta reflexión:

“Enfermería ha perdido su alma por servir ya sea a la medicina, la tecnología y la práctica basada en la evidencia, las que por cierto se necesitan; pero sin la dimensión humana, esto puede ser destructivo. Es fundamental preservar la esencia humana y reposicionar el cuidado dentro de nuestros sistemas" (18). 


\section{> Referencias bibliográficas}

1. Real Academia de la Lengua Española. Diccionario. [Actualizado 2017; Consultado abr 2017]. Disponible en: http://dle.rae.es/

2. Oliveira M, Fenili R, Zampieri M, Martins C. Un Ensayo sobre la comunicación en los cuidados de enfermería utilizando los sentidos. Enferm Global [en línea]. 2006 (may). [Consultado 19 abr 2017]: 8. Disponible en: revistas.um.es/eglobal/article / view/450

3. Vanegas B, Castro L, Páez M, Ramírez N, Salcedo L. Comunicación del profesional de enfermería en pacientes que tienen dificultad en la expresión verbal por sordera. Rev Colomb Enferm. 2008; $3(3): 13-20$

4. Landman C, Cruz MJ, García E, Pérez P, Sandoval P, Serey K, et al. Satisfacción usuaria respecto a la competencia de comunicación profesional de enfermería. Cienc enferm. 2015; 21(1): 91-102.

5. Galiano M, Falcon O, González L, Montesino I, Capote J, Fleitas H. Comunicación del personal de enfermería con el paciente glaucomatoso. Invest Medicoquir. [Internet]. 2014 (jul-dic) [Consultado 05 may 2017]; 6 (2): 229-41. Disponible en: http:// www.medigraphic.com/pdfs/invmed/cmq-2014/ cmq142g.pdf

6. Raile M, Marriner A. Modelos y teorías de enfermería. 8va ed. Barcelona (España): Elsevier; 2014.744p.

7. Müggenburg C, Robles R, Valencia A, Hernández $M$, Olvera $S$, Riveros R. Evaluación de la percepción de pacientes sobre el comportamiento de comunicación del personal de enfermería: diseño y validación en población mexicana. Salud Ment. 2015; 38(4): 272-80.
8. Rousseau JJ. Ensayo sobre el origen de las lenguas. Buenos Aires (Argentina): Ediciones Godot; 2014. 112p.

9. Bengoa J. Historia del pueblo mapuche siglos XIX y XX. 3ra ed. Santiago de Chile: Ediciones LOM. 2000. 430p.

10. Barranco S, Ventura P, Coronado P. Estrategias de comunicación de los profesionales de enfermería con personas sordas o ciegas. Index Enferm. 2016; 25(4): 253-7.

11. Fuentes JL. Comunicación: Estudio del lenguaje. 2da ed. Barcelona (España): Bibliográfica Internacional. 2007. 554p.

12. Laddy S, Pepper JM. Bases conceptuales de la enfermería profesional. Ciudad de México: JB Lippincott company. 1989. 395p.

13. González C. Competencias en enfermería intensiva. Madrid (España); Editorial Académica Española. 2016. 142 p.

14. Alles M. Diccionario de competencias laborales. Buenos Aires (Argentina); Ediciones Gránica SA. 2004. 296p.

15. Mendonça LC, De Freitas KN, Pereira K, Ferreira T. Comunicação entre a equipe de enfermagem e familiares de pacientes em unidade de terapia intensivo. Cul cuid. 2014 (may-ago); XVIII(39): 84-92.

16. Ramírez P, Müggenburg C. Relaciones personales entre la enfermera y el paciente. Enferm universitaria. 2015 (sep); 12(3): 134-43.

17. Superintendencia de Salud de Chile. Estudio de usuarios del sistema de salud y prestadores respecto a la ley de derechos y deberes del paciente. Santiago de Chile; 2014. 102 p. 
18. Urra M, Jana A, García M. Algunos aspectos esenciales del pensamiento de Jean Watson y su teoría de cuidados transpersonales. Cienc enferm. 2011; 17(3): 11-22.

19. Ferreira S, Oliveira L, Munari D, Barbosa M, Peixoto M, Ayres K, et al. Cuando la comunicación es nociva en el encuentro entre profesional y familia del niño hospitalizado. Enferm glob. [Internet]. 2015 (ene). [Consultado 28 may 2017] ; 14(37): 192-203. Disponible en: http://scielo.isciii. es/scielo.php?script=sci_arttext \&pid=S169561412015000100009\&lng=es.

20. Díaz A, De Vera I, Del Toro M, Bilbao N, Vega A. Modelo de Estructura Funcional para el Cuidado y Comunicación a Pacientes Pediátricos. Investigación cualitativa en educación [Internet]. 2016. [Consultado 28 mayo 2017]; 2. Disponible en: http://proceedings.ciaiq.org/index.php/ ciaiq2016/article/view/749/736

21. Miralles M. Lenguaje y comunicación. 10a Ed. Chile. Ed. Universidad Católica de Chile. 2006. $387 p$.

22. Landman C, Canales S, Garay V, García P, López C, Pérez J, et al. Cuidado humanizado desde la perspectiva del enfermero de emergencia en un hospital de Quillota, Chile. Enfermería (Montev). 2014; 3(2): 12-21.

23. Hernández L. La mujer con cáncer de mamas: una experiencia desde la perspectiva del cuidado humano. Enferm universitaria. 2016 (sep); 13(4): 253-9.

24. Landman A, Del Alcazar RM, Madrid Y, Pais L, Rosenkranz E, Vivanco I. Habilidades para el cuidado humanizado de estudiantes de enfermería. Enfermería (Montev). 2014; 5 (1): 29-34.

25. Rivera S. El arte de cuidar en enfermería. Horiz enferm. 2004; 15: 11-22. 